\title{
L-Dopa and amantadine hydrochloride in extra-pyramidal disorders
}

\author{
J. D. PARKeS \\ M.D., M.R.C.P.
}

\author{
R. P. KNILL-JONES \\ M.B., M.R.C.P.
}

\author{
P. J. Clements \\ M.B. \\ Department of Neurology, King's College Hospital, London, S.E.5
}

\begin{abstract}
Summary
L-Dopa and amantadine hydrochloride were separately given to patients with diverse extra-pyramidal disorders including progressive supranuclear palsy; porto-systemic encephalopathy; oculogyric crises; drug-induced Parkinsonism; Huntington's chorea, hemiballismus; spasmodic torticollis, familial tremor; and athetosis. Akinesia and rigidity were improved in the first two conditions and simultaneously there was improvement in disordered eye movement. Amantadine provoked an exacerbation in spasmodic torticollis and familial tremor. No other condition was influenced. Both amantadine and L-dopa will facilitate eye movement mechanisms: it seems probable that both drugs act on dopamine-sensitive areas within the CNS. Neither drug will give significant benefit in the above disorders.
\end{abstract}

\section{Introduction}

In addition to improvement in Parkinsonism, L-dopa will correct akinesia and dystonia in manganese intoxication (Mena et al., 1970), akinesia, rigidity and abnormal eye movements in progressive supranuclear palsy (Mendell, Chase \& Engel, 1970), akinesia and rigidity in juvenile Huntington's chorea (Barbeau, 1969), and motor performance, tremor and akinesia in Wilson's disease (Barbeau \& Friesen, 1970).

These disorders have little in common except for disease of the extra-pyramidal system, but it seems probable that $\mathrm{L}$-dopa is active in all by increasing the cerebral dopamine concentration. Amantadine, although having no close chemical relationship with dopamine or other neuro-transmitters, causes improvement in Parkinson's disease. Amantadine produces atropine-like side effects but resembles dopa- mine rather than drugs such as benzhexol in its therapeutic spectrum (Parkes et al., 1970). Amanta- $\overrightarrow{-}$ dine causes monoamine release from neurongl storage sites (Grelak et al., 1970). Thus increased dopamine (or serotonin) may be available for neum $\vec{T}-\vec{T}$ transmission. These facts led us to compare theo action of L-dopa and amantadine in patients suffet $-\frac{C}{D}$ ing from diverse extra-pyramidal disorders, 虾 the majority of which degenerative changes present in brain areas with high monoamine con -1 centration. It was hoped to define the clinical valöe of amantadine in such disorders and at the sameo time gain further knowledge as to its mode of action.

\section{Case reports}

Progressive supranuclear palsy

1. A 61-year-old man complained of unsteadiness and impairment of memory of 1 year's duration. WAIS assessment gave a performance IQ of 78 and a verbal IQ of 85 . There was a slight nominal 을 dysphasia, speech being of low volume and dysarthric. He was most unsteady whilst walking, although 3 the posture was erect. There was considerable impairment of facial expression, great difficulty in 3 commencing movements and increased resistance to 0 passive movements of the neck and limb muscles. No tremor was present. Volitional and pursuit $\frac{D}{0}$ conjugate eye movements were absent both on vertical and lateral gaze, apart from limited volun- $N$ tary movement of the eyes to the left. Full vertical $N$ eye movement accompanied neck flexion and $N$ extension.

2. A 62 -year-old man was seen with a 3 year history of slurred speech, slowness and clumsiness of movement. This patient had a verbal IQ of 88 and $\stackrel{\oplus}{\oplus}$ 


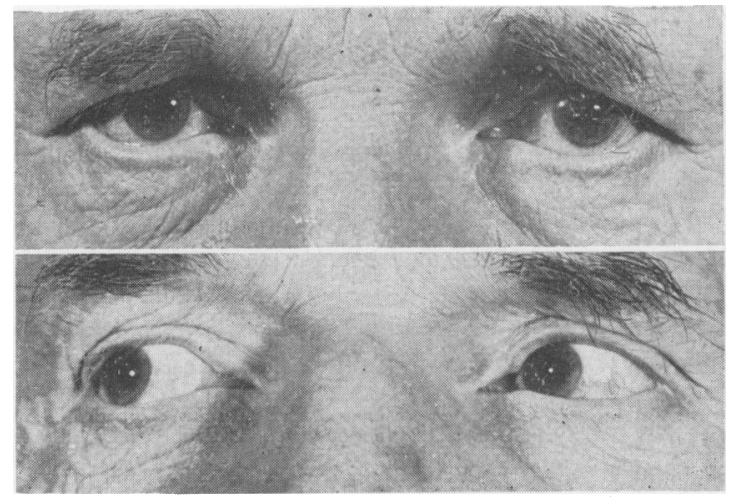

Fig. 1. The eye movements in the first patient with supranuclear palsy. On each occasion the patient is attempting voluntary right lateral gaze with the head held stationary. The upper figure shows the paralysis of gaze prior to treatment and the lower figure the voluntary gaze possible following 1 week's treatment with amantadine $300 \mathrm{mg} /$ day.

performance IQ of 93, showing some learning difficulty. Speech was a little dysarthric but not difficult to understand. Posture was erect with no trunk or limb flexion; however gait was considerably impaired with frequent stumbles and great difficulty in turning. No tremor was seen. There was considerable increase in resistance to passive movements of the limb muscles. Voluntary and pursuit conjugate vertical eye movements were lost but lateral gaze was present although restricted in range. Full vertical eye movement accompanied neck flexion and extension.

Both these patients were given L-dopa $(4 \mathrm{~g} /$ day and $6 \mathrm{~g} /$ day respectively). The responses to $\mathrm{L}$-dopa were comparable in both patients with a moderate but not dramatic improvement in akinesia and rigidity. There was a slight increase in range of voluntary lateral, but not vertical conjugate eye movements in both cases. IQ assessment remained unchanged. L-dopa treatment was stopped in both patients after 2 weeks, being replaced by amantadine $300 \mathrm{mg} /$ day. This also resulted in an improvement in speed of movement with less rigidity of limb muscles and an increased range of voluntary lateral eye movements. Further tests of memory showed no change in mental function in either patient, in both of whom amantadine and L-dopa did not produce sufficient improvement to warrant long term treatment.

\section{Porto-systemic encephalopathy}

A 52-year-old-man with advanced hepatic cirrhosis developed variable confusion with impairment of concentration. He had loss of facial expression, impairment of rapid finger movements, and a slow

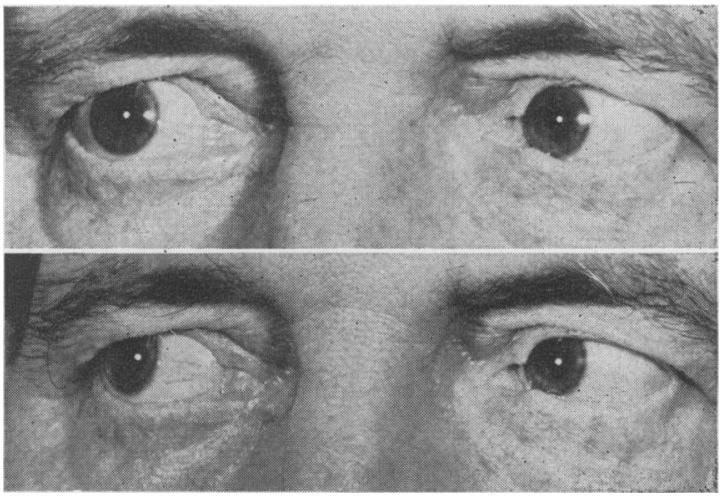

FIG. 2. The eye movements of the patient with portosystemic encephalopathy. The upper figure shows the convergence spasm accompanying right lateral gaze before treatment and the lower figure shows the reduction in this following amantadine treatment.

gait with great unsteadiness on turning. These features were attributed to porto-systemic encephalopathy, and showed some variation with treatment. In addition, he developed postural vertigo and a profound impairment of eye movements. Volitional or pursuit gaze in a vertical or horizontal direction was prevented by involuntary over-convergence of the eyes. These latter features were attributed to a brain stem vascular lesion. Posture was normal, no tremor was present and muscle tone was unremarkable.

This patient was given amantadine $300 \mathrm{mg} /$ day with improvement in gait, facial expression and finger dexterity. There was also considerable recovery of eye movement, although ocular convergence still occurred after prolonged upward gaze. The patient felt better in himself but no change in postural vertigo or mental function occurred. He was able to detect amantadine substitution by placebo (although not told of this) and returned to his former condition, eye movements deteriorating. The liver disorder was progressive and we were unable to give L-dopa under conditions comparable with amantadine.

\section{Oculogyric crises}

A 56-year-old man with post-encephalitic Parkinsonism suffered frequent oculogyric crises, with upward deviation of the eyes accompanied by forced eye closure. Following amantadine $500 \mathrm{mg} /$ day, these spasms became much more frequent although of shorter duration. L-Dopa ( $3 \mathrm{~g} /$ day) gave considerable relief in akinesia and rigidity, but did not change either the frequency or duration of involuntary eye movements.

\section{Phenothiazine-induced Parkinsonism}

A 62-year-old lady was referred with Parkinsonism 
of 5 years duration. She denied any drug intake. Examination showed considerable akinesia and rigidity but no tremor. This patient was not helped by L-dopa $4 \mathrm{~g} /$ day for 2 weeks, following which she was given amantadine $300 \mathrm{mg} /$ day, again with no improvement. She later admitted to taking trifluoperazine regularly during the preceding 5 years. This drug was stopped and all Parkinsonian features disappeared in the following 2 months.

\section{Huntington's chorea}

Four female patients with this disorder, all with profound mental deterioration and very disabling chorea were given amantadine hydrochloride 300 $\mathrm{mg} /$ day. No change in mental state or movement disorder was seen. These patients were then given L-dopa, individual doses varying from $2 \mathrm{~g}$ /day to $6 \mathrm{~g} /$ day, but no improvement was found after a 1 month period, choreiform movements being unaltered.

\section{Hemiballismus}

A 78-year-old lady suddenly developed abrupt rapid and extensive movements of the right arm and leg. She showed uncontrollable hemiballismus, being unable to walk unaided. There was a slight improvement in this patient's involuntary movements when given amantadine $300 \mathrm{mg} / \mathrm{day}$, but this improvement continued on stopping treatment and the patient was not given L-dopa.

\section{Spasmodic torticollis}

A 48-year-old man developed involuntary movements of the head and neck which with considerable effort he could momentarily control. This varied in severity over a 5 year period. After 2 days of amantadine treatment $(300 \mathrm{mg} /$ day $)$ there was a very considerable exaggeration of both the frequency and degree of head movement and he became distressed. On stopping amantadine within $24 \mathrm{hr}$ he recovered his former state. L-Dopa $4 \mathrm{~g}$ /day had no effect on neck movement after a 1 month period.

\section{Familial tremor}

Two female patients, aged 53 and 56, with a slight tremor of the arms and head, both with a family history of tremor, were given amantadine $300 \mathrm{mg} /$ day. Both showed a marked exacerbation of tremor which subsided on stopping amantadine. In neither case did L-dopa (3 g/day:1.5 g/day) affect the tremor.

\section{Athetosis}

A man of 40 with cerebral diplegia was seen with considerable spasticity of all limbs and movements of a predominantly athetoid nature. There was a profound mental retardation and speech was extremely dysarthric. He made no response to eithe L-dopa $4 \mathrm{~g}$ /day or amantadine $300 \mathrm{mg} /$ day, botho drugs being given for a 2 week period.

Abnormal movements produced by L-dopa and aman $\frac{2}{2}$ tadine

In thirty-nine patients treated with L-dopa, choreiform movements have been seen in four and abnormal movements of the neck or jaw seen in two 흠 respiratory tics in a further two. Similar movements have not been seen in eighty-nine patients given amantadine up to $500 \mathrm{mg} /$ day, although one suches patient reported uncontrollable jerking movementsof the legs on waking in the morning.

\section{Discussion}

The considerable variation from day to day in theo clinical features of the conditions we describe makes. the response assessment difficult. However, there seemed no doubt of improvement of eye movementr and akinesia in the patients with progressive supra + nuclear palsy and liver disease and this was confirmed by ciné films. The increased tremor in the patients with familial tremor, exacerbation of movement fip spasmodic torticollis, and increase in frequency ${ }^{-}$ oculogyric crises exceeded normal variation.

There was a striking similarity between the action of L-dopa and amantadine in porto-systemic 손 cephalopathy and progressive supranuclear palsy. Pathological changes are widespread in both (Victor, Adams \& Cole, 1965; Behrman et al., 1969) (sit akinesia is probably secondary to involvement of substantia nigra and its connections. Neither drug affected the mental disorder over a short period.

Dopamine is the predominant catecholamine ing the substantia nigra and the pallidum (Hornykie $\frac{1}{2}$ wicz, 1966) and these areas are amongst those mos severely involved in progressive supranuclear palsys (Behrman et al., 1969). The return of eye movement produced by L-dopa in this condition suggests that these reduced eye movements may be associate ${ }_{5}$ with disordered function of the substantia nigra o르. pallidum, this gaze palsy being thus of extra pyramidal rather than pyramidal origin. Amanta 3 dine acted like L-dopa, a finding in keeping with the concept that amantadine acts by releasing dopamine at nerve terminals.

The pathogenesis of oculogyric crises is not known but this involuntary spasm stands in contrast to the paralysis of voluntary gaze of progressive supra nuclear palsy. Oculogyric crises were aggravated by amantadine and it is noteworthy that $\mathrm{L}$-dopa mayalso provoke oculogyric spasm as in Case 1 of Cotzias Papavasiliou \& Gellene (1969), and alsocaused oculog gyric crises in a case of Wilson's disease (Barbeau \& Friesen, 1970). These facts suggest a common site and mode of action of both L-dopa and amantadine 
in the facilitation of conjugate eye movement mechanisms, probably at extrapyramidal level.

Phenothiazine-induced Parkinsonism has been attributed to a receptor blocking action of phenothiazines on CNS catecholamine transmission (Brodie, Spector \& Shore, 1959). Dopa does not improve phenothiazine-induced Parkinsonism (McGeer et al., 1961) and it is not surprising that L-dopa and amantadine also had no effect. Neither drug was of value in the treatment of chorea or athetosis and hemiballismus was probably not improved by amantadine. This suggests that these movement disorders are not primarily provoked by failure of dopaminergic transmission and this view is supported by the provocation of these movements by L-dopa. These movements have not been seen with amantadine and this may be secondary to an exhaustion of dopamine from synaptosomes produced by amantadine, whilst L-dopa may lead to an excess saturation of synaptic regions with dopamine.

The increased movement in spasmodic torticollis and familial tremor seen with amantadine, but not L-dopa, could be related to the hyperactive state sometimes caused by amantadine when given in high dosage (Vernier et al., 1969).

These clinical findings suggest that L-dopa and amantadine are only of value in extra-pyramidal disease primarily affecting the substantia nigra and striatum. Other conditions characterized by involuntary movement are not helped by either drug.

The effects of both drugs on eye movements needs to be further explored. The similarity of therapeutic spectra of the two drugs is in favour of the suggestion that amantadine causes dopamine release at nerve terminals, although the possibility remains that amantadine may influence serotonin rather than dopamine, or act as a 'false transmitter' within the CNS.

\section{References}

BARBEAU, A. (1969) L-Dopa and juvenile Huntington's disease. Lancet, ii, 1066.

Barbeau, A. \& Friesen, H. (1970) Treatment of Wilson's disease with L-dopa after failure with penicillamine. Lancet, i, 1180.

Behrman, S., Carroll, J.D., Janota, I. \& Matthews, W.B. (1969) Progressive supranuclear palsy. Clinico-pathological study of four cases. Brain, 92, 663.

Brodie, B.B., Spector, S. \& Shore, P.A. (1959) Interaction of drugs with norepinephine in the brain. Pharmacological Reviews, 11, 548.

Cotzias, G.C., Papavasiliou, P.S. \& Gellene, R. (1969) Modification of Parkinsonism-chronic treatment with L-dopa. New England Journal of Medicine, 280, 337.

Grelak, R.P., Clark, R., Stump, J.M. \& Vernier, V.G. (1970) Amantadine-dopamine interaction. Science, 169, 203.

HoRnykiewicz, O. (1966) Dopamine (3-hydroxytyramine) and brain function. Pharmacological Reviews, 18, 925.

McGeer, P.L., Boulding, J.E., Gibson, W.C. \& Foulkes, R.G. (1961) Drug-induced extrapyramidal reactions. Journal of the American Medical Association, 177, 665.

Mena, I., Court, J., Fuenzalida, S., Papavasiliou, P.S. \& Cotzias, G.C. (1970) Chronic manganese poisoning. Treatment with L-dopa or 5-OH tryptophane. New England Journal of Medicine, 282, 5.

Mendell, J.R., Chase, T.N. \& Engel, W.K. (1970) Modification by L-dopa of a case of progressive supranuclear palsy. Lancet, i, 593.

Parkes, J.D., Zilkha, K.J., Knill-Jones, R.P., Clements, P.J. \& BAXTER, R. (1970). Comparative trial of L-dopa and amantadine hydrochloride in the treatment of Parkinsonism. International Journal of Experimental Pharmacology and Therapeutics. (In press.)

Vernier, V.G., Harmon, J.B., Stump, J.M., Lynes, T.E., Marvel, J.P. \& SMith, D.H. (1969) The toxicologic and pharmacologic properties of amantadine hydrochloride. Toxicology and Applied Pharmacology, 15, 642.

Victor, M., Adams, R.D. \& Cole, M. (1965) The acquired (non-Wilsonian) type of chronic heptatocerebral degeneration. Medicine (Baltimore), 44, 345. 